\title{
THE WORKSHOPS ON STOCHASTIC GEOMETRY, STEREOLOGY AND IMAGE ANALYSIS
}

\author{
Eva B. VEDEL JENSEN ${ }^{\bowtie, 1}$ AND HANS JøRGEN G. Gundersen ${ }^{2}$ \\ ${ }^{1}$ Department of Mathematics, Aarhus University, Ny Munkegade 118, DK-8000 Aarhus C, Denmark; ${ }^{2}$ Section \\ for Stereology and Microscopy, Danish Neuroscience Centre, Aarhus University Hospital, Building 10G, \\ Nørrebrogade 44, DK-8000 Aarhus C, Denmark \\ e-mail: eva@math.au.dk, stereohj@gmail.com \\ (Received April 22, 2017; revised August 3, 2017; accepted August 23, 2017)
}

\begin{abstract}
The workshops on stochastic geometry, stereology and image analysis have been held every second year since 1981. The first workshops were small meetings, but with prominent speakers. Over the years, the workshops have increased in size and in impact too. Nowadays, the workshops have developed into the main occasion to promote recent advances in stochastic geometry, stereology and image analysis.
\end{abstract}

Keywords: biannual workshops, education of researchers, honouring of colleagues, interaction with neighbouring fields, pioneers, satellite courses.

\section{INTRODUCTION}

This paper gives an overview of the series of workshops on stochastic geometry, stereology and image analysis.

These biannual workshops, arranged since 1981, have had a number of important roles in relation to the development of the stochastic geometry community. First of all, the workshops have provided a broad forum for communicating and discussing recent advances in stochastic geometry, stereology and image analysis. Secondly, the workshops have nurtured young talents in our field. By interaction with prominent speakers from neighbouring fields, the workshops have also contributed to new directions of stochastic geometry as well as the present day standing of stochastic geometry in mathematics, probability theory and statistics.

During the time span of the workshops, stochastic geometry, stereology and image analysis have developed into established disciplines. Nowadays, they provide valuable tools in a number of fields, including life sciences, geology, materials science and engineering.

Below, we give an account of all these aspects, but for obvious reasons we cannot include all available material.

The paper is organized as follows. First, we give a short account of the workshop history, including venues, organizers, participants, etc. Secondly, we describe some of the topics dealt with at the workshops, thereby giving an impression of the scientific impact of the workshops. Finally, we discuss the possible future role of these workshops. The paper ends with a selection of important monographs on stochastic geometry, stereology and image analysis, published in recent years. In the Appendix, we give an overview of topics, presented in workshop talks.

\section{WORKSHOP HISTORY}

The venue and organizers of the complete list of workshops, covering the period 1981-2017, are shown in Table 1.

During the years, the workshop titles have varied slightly. For instance, 'image analysis' was not included in the titles of the very early workshops.

Prominent speakers have taken part in the workshops from the very beginning, including the pioneers of modern stereology and stochastic geometry, Roger Miles and Joseph Mecke. Over the years, the workshops have increased in size and in impact too. The number of participants has been growing steadily, from 10-15 participants in the earliest days till about 45 participants in 1995 and more than 70 at the most recent workshops.

The initiation of this workshop series was motivated by the new approaches to stereology, pioneered by Roger Miles, that appeared in the late 1970s and the early 1980s. At the early workshops, many of these important advances in stereology were reported. During the 1980s, it was difficult for researchers from the Eastern Europe block to participate in the workshops. It was indeed an exception that Joseph Mecke was able to participate 


\begin{tabular}{rrll}
\hline & Year & Venue & Organizers \\
\hline 1 & 1981 & Aarhus & Gundersen, Jensen \\
2 & 1983 & Aarhus & Gundersen, Jensen \\
3 & 1985 & Bath & Baddeley \\
4 & 1987 & Bern & Cruz-Orive, Weibel \\
5 & 1989 & Amsterdam & Baddeley \\
6 & 1991 & Oberwolfach & Baddeley, Jensen, Stoyan, Weil \\
7 & 1993 & Valencia & Montes \\
8 & 1995 & Sandbjerg & Gundersen, Jensen \\
9 & 1997 & Comillas & Cruz-Orive \\
10 & 1999 & Calgary & Enns \\
11 & 2001 & Perth & Baddeley, Nair \\
12 & 2003 & Prague & Beneš, Rataj, Saxl, Jańaček, Pawlas, Prokešová \\
13 & 2005 & Bern & Molchanov \\
14 & 2007 & Neudietendorf & Nagel \\
15 & 2009 & Blaubeuren & Schmidt, Spodarev \\
16 & 2011 & Sandbjerg & Hahn, Kiderlen \\
17 & 2013 & Toruń & Jakubowski, Ziemkiewicz \\
18 & 2015 & Lingen & Reitzner, Thäle \\
19 & 2017 & Luminy & Calka, Coeurjolly, Coupier, Estrade, Molchanov \\
\hline
\end{tabular}

Table 1. The complete list of workshops.

in the 2nd workshop organized 1983 by us in Denmark. "Die Wende", the great revolution in Eastern Europe, implied that the restriction in participation in the workshops from 1991 and onwards was no longer there. As a consequence, the number of participants increased and the topics widened and included now stochastic geometry in general and spatial point processes in particular. During the 1990s, the French school with Georges Matheron and Jean Serra influenced the development of second-order stereology, as reflected in many talks at the workshops during this time period. In the last decades, the possibilities offered by new microscopes and advanced image analysis have been the focus in a number of workshop talks.

The number of $\mathrm{PhD}$ students and postdocs attending the workshops has grown during the years, and a large poster session with informal discussions between junior and senior researchers is now a fixed element of the workshops. At some of the latest workshops, satellite courses have been arranged. For example, a course on analysis of spatial point patterns was given at the 16th workshop, organized 2011 by Ute Hahn and Markus Kiderlen in Sandbjerg, and at the 18th workshop, organized 2015 by Matthias Reitzner and Christoph Thäle in Lingen, an introductory course on stochastic geometry was arranged.

The venues have been in Europe, except at two occasions: the 10th workshop organized 1999 by Ernest Enns in Calgary, Canada, and the 11th workshop organized 2001 by Adrian Baddeley and Gopalan Nair in Perth, Australia.

Many disciplines have always been represented at these workshops. The reason is, of course, that stochastic geometry, stereology and image analysis have so wide-spread applications. Disciplines dealt with at the workshops include astronomy, biology, communication networks, forestry, genetics, materials science, medicine and physics. Another characteristic feature of the workshops has been the willingness to learn from related fields by inviting prominent speakers from such fields.

The main purpose of the workshops has been to promote recent advances in stochastic geometry, stereology and image analysis. But the workshops have also provided a forum for honouring our colleagues. This happened at the 13th workshop, organized 2005 by Ilya Molchanov in Bern, where the scientific achievements of the grand-old-man of stochastic geometry, Dietrich Stoyan, were celebrated. Likewise, a memorial session for Tomasz Schreiber (1975-2010) was arranged at the 17th workshop, organized 2013 by Adam Jakubowski and Bartosz Ziemtiewicz in Toruń.

During the time span of the workshops, further conferences and meetings have been organized in the same topics. In the 1980s, the East German point process school arranged the Georgenthal meetings in Thuringia. A number of researchers from outside Eastern Europe participated in these meetings and benefited from the direct exposition to the spatial point 


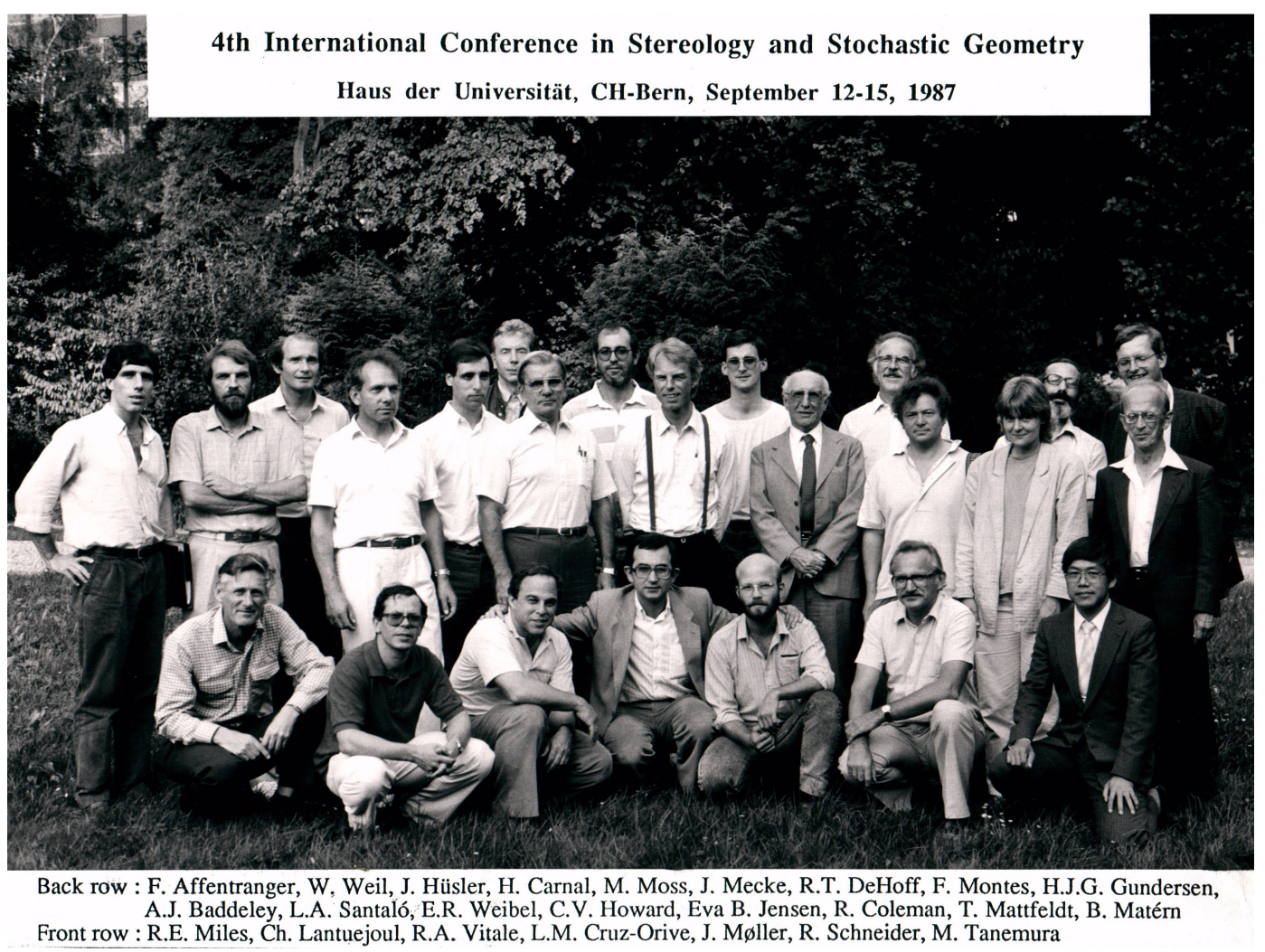

Fig. 1. The famous group photo from the 4th Workshop on Stochastic Geometry, Stereology and Image Analysis, Bern, 1987, organized by Luis Cruz-Orive and Ewald Weibel. Note that 'image analysis' was not part of the official title of this workshop. Many of the researchers on this photo participated in the following workshops, including the pioneers of modern stereology and stochastic geometry, Roger Miles and Joseph Mecke.

process approach of our East German colleagues. In Czechoslovakia and later in the Czech Republic, a series of conferences on stereology, spatial statistics and stochastic geometry was initiated in the late 1970s. These conferences are still arranged every sixth year and are now called S4G conferences.

\section{SCIENTIFIC IMPACT OF THE WORKSHOPS}

We believe that one of the reasons why the workshops have been such a success is the wide range of research problems, presented and discussed at the workshops. The workshops have provided a very broad forum for communicating and discussing recent advances.

In the Appendix, we give a selection of topics, presented in workshop talks. The topics are grouped under well-known headings from stochastic geometry. The list gives an impression of the richness of the research problems, taken up at the workshops.

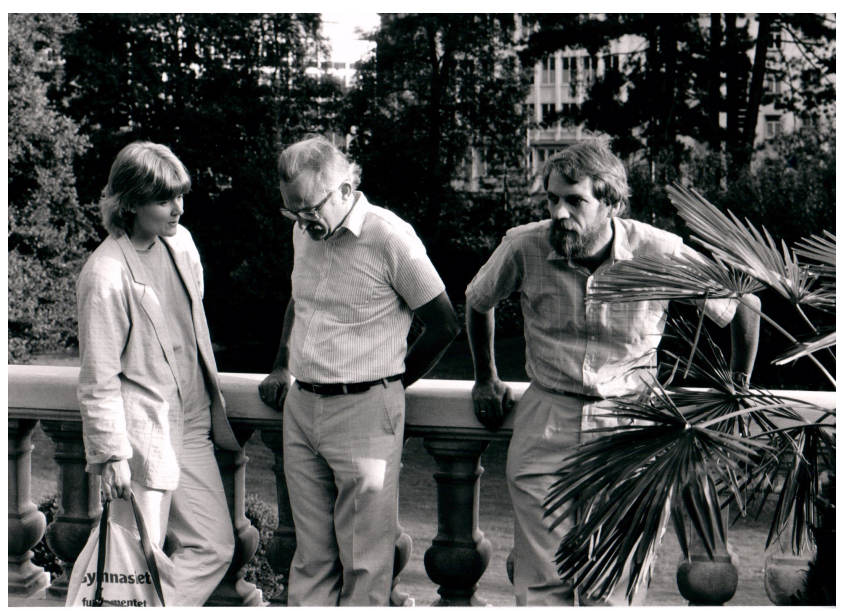

Fig. 2. Discussion concerning Blaschke-Petkantschin formulae at the 1987 workshop. Later, these formulae developed into an important tool in rotational integral geometry and local stereology.

During the whole time period, covered by the workshops, mathematical disciplines such as integral geometry and geometric measure theory have had a prominent position in the scientific programme. In the last decade, the workshops have been enriched by 


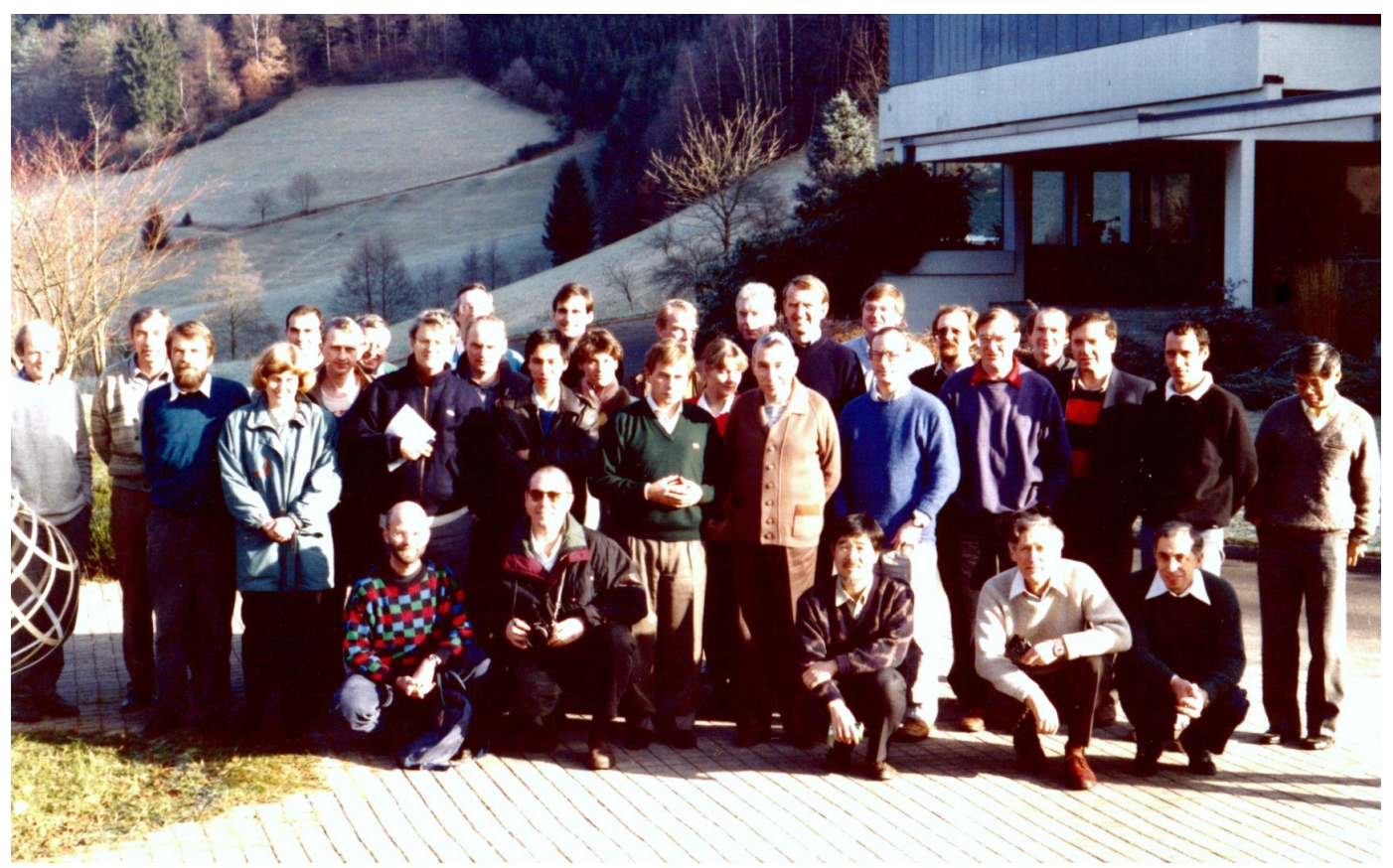

Fig. 3. Group photo from the 6th Workshop on Stochastic Geometry, Stereology and Image Analysis, Oberwolfach, 1991, organized by Adrian Baddeley, Eva B. Vedel Jensen, Dietrich Stoyan and Wolfgang Weil.

participation of Andreas Bernig, Frankfurt (algebraic integral geometry) and Semyon Alesker, Tel Aviv (valuations and integral geometry).

Tools from modern probability theory have played an increasingly important role at the workshops. In a number of talks during the last decade or so, new results have been presented on secondorder properties, asymptotics and, more generally, distribution theory for a number of stochastic geometry estimators.

At the workshops, it has been a continuous theme to transfer statistical inference methods from classical and computational statistics to stochastic geometry models. Important topics are here pseudolikelihood, Palm likelihood, Bayesian inference, simulation based inference and spatial residuals.

The workshops have covered the development of modern stereology that differs from classical stereology by having rigorous statistical foundations and a much wider range of stereological identities. During the very first workshops, methods for analyzing particle populations with not necessarily convex particles were developed and stereology was enriched by a number of new sampling designs. In a modelbased setting, these designs induced new stochastic geometry models with different types of invariance properties. In recent years, stereology of tensors have been developed.

A range of image modalities has been discussed at the workshops, including computed tomography, functional magnetic resonance imaging (fMRI), scanning electron microscopy and synchroton microtomography. New analysis tools for digital images have been developed, using stochastic geometry models. Examples are segmentation of digital images, using Markov random fields, and Bayesian analysis of fMRI, using spatial point processes as priors.

At the workshops, there have always been reports on applications of stochastic geometry, stereology and image analysis, especially from biology and materials science. The applications have covered the full range of scales from microscopy data via forestry data to astronomy data, revealing the spatial distribution of galaxies. During the last decade, tensor analysis has been introduced in biology and physics for analysis of spatially structured materials. Another application area of stochastic geometry that is becoming increasingly important is telecommunications networks.

\section{THE FUTURE}

We expect that the workshops will continue to be a main forum for communicating and discussing recent advances in stochastic geometry, stereology and image analysis.

At the workshops, stochastic geometry will be nurtured by inputs from mathematics, probability theory and statistics, and, at the same time, challenged 


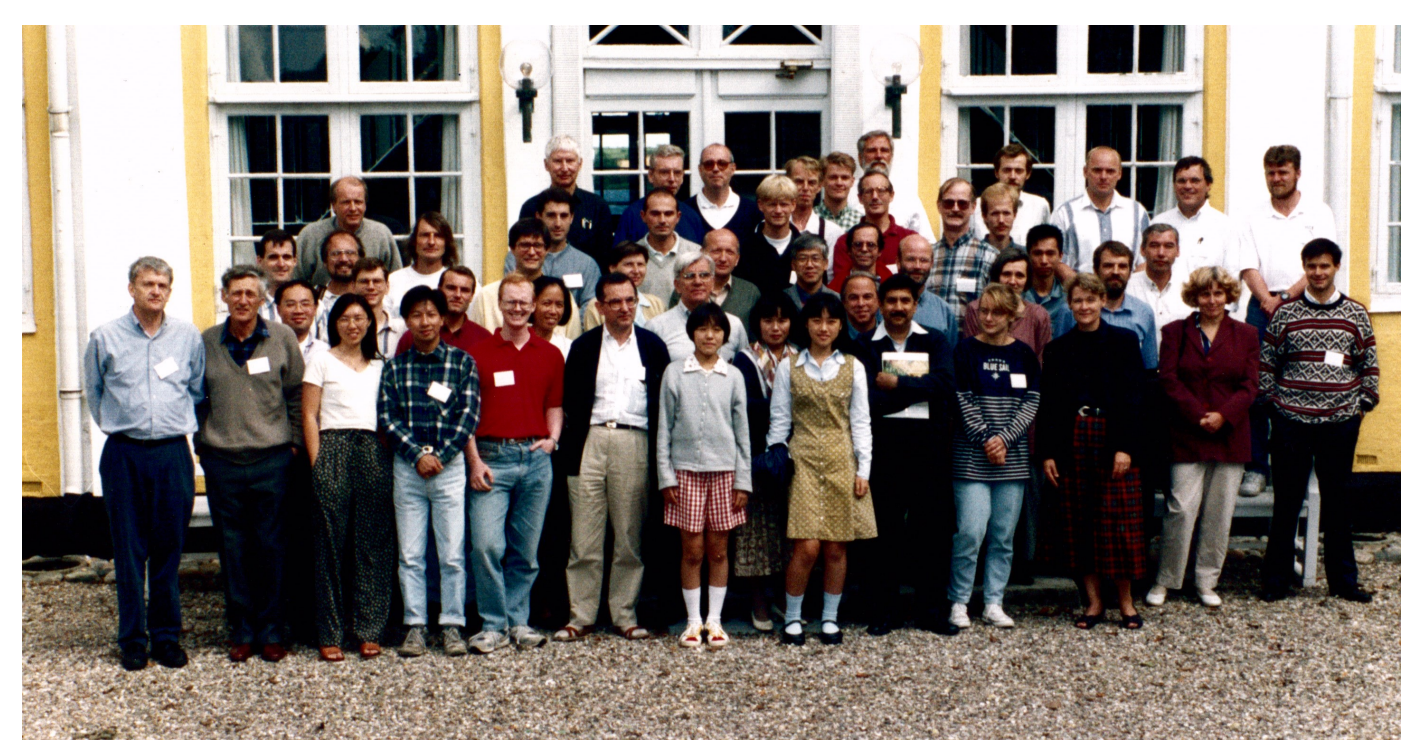

Fig. 4. Group photo from the 8th Workshop on Stochastic Geometry, Stereology and Image Analysis, Sandbjerg, 1995, organized by the authors. The abstracts from this workshop were published in Advances in Applied Probability, which was also the case for the abstracts from the 9th workshop, organized 1997 by Cruz-Orive in Comillas.

by the increasing number of research questions from applications.

We also expect that talks about stereology and image analysis will be an integral part of the workshops in the future. Stereology is an efficient tool for estimating 3D global parameters from sections. In contrast, more complex parameters like shape, arrangement or size distribution are often best obtained by 3D image analysis. However, stereology of tensors might become a useful alternative to the direct $3 \mathrm{D}$ analysis in biological applications.

\section{ACKNOWLEDGEMENTS}

This research was supported by Centre for Stochastic Geometry and Advanced Bioimaging, funded by a grant from the Villum Foundation.

\section{A SELECTION OF RECENT MONOGRAPHS}

Baddeley A, Jensen EBV (2005). Stereology for statisticians. Boca Raton: Chapman \& Hall/CRC.

Beneš V, Rataj J (2004). Stochastic geometry: selected topics. Boston: Kluwer Academic Publishers.

Chiu SN, Stoyan D, Kendall WS, Mecke, J (2013). Stochastic geometry and its applications. 3rd ed. Chicester: John Wiley and Sons,

Kendall WS, Molchanov I, eds (2010). New perspectives in stochastic geometry. Oxford: Oxford University Press.

Molchanov I (2005). Theory of random sets. London: Springer-Verlag.
Møller J, Waagepetersen RP (2004). Statistical inference and simulation for spatial point processes. Boca Raton: Chapman \& Hall/CRC.

Nguyen HT (2006). An introduction to random sets. Boca Raton: Chapman \& Hall/CRC.

Ohser J, Mücklich F (2000). Statistical analysis of microstructures in materials science. Chicester: John Wiley and Sons.

Ohser J, Schladitz K (2009). 3D images of materials structures: processing and analysis. Weinheim: Wiley$\mathrm{VCH}$.

Schneider R, Weil W (2008). Stochastic and integral geometry. Berlin: Springer-Verlag.

\section{APPENDIX: WORKSHOP TALKS}

In this Appendix, we give a selection of topics, presented in workshop talks. The topics are grouped under well-known headings from stochastic geometry. The list is only a selection, but still, it gives an impression of the variety of topics, discussed at the workshops.

- Boolean models: compact grains, non-stationarity, statistics of Boolean models, tensor density formulae, topology

- Integral geometry/geometric measure theory: Blaschke-Petkantschin formulae, generalised normal measures, hyperbolic integral geometry, integral formulae for support functions, isotropic projections, non-convex bodies, rotational integral 


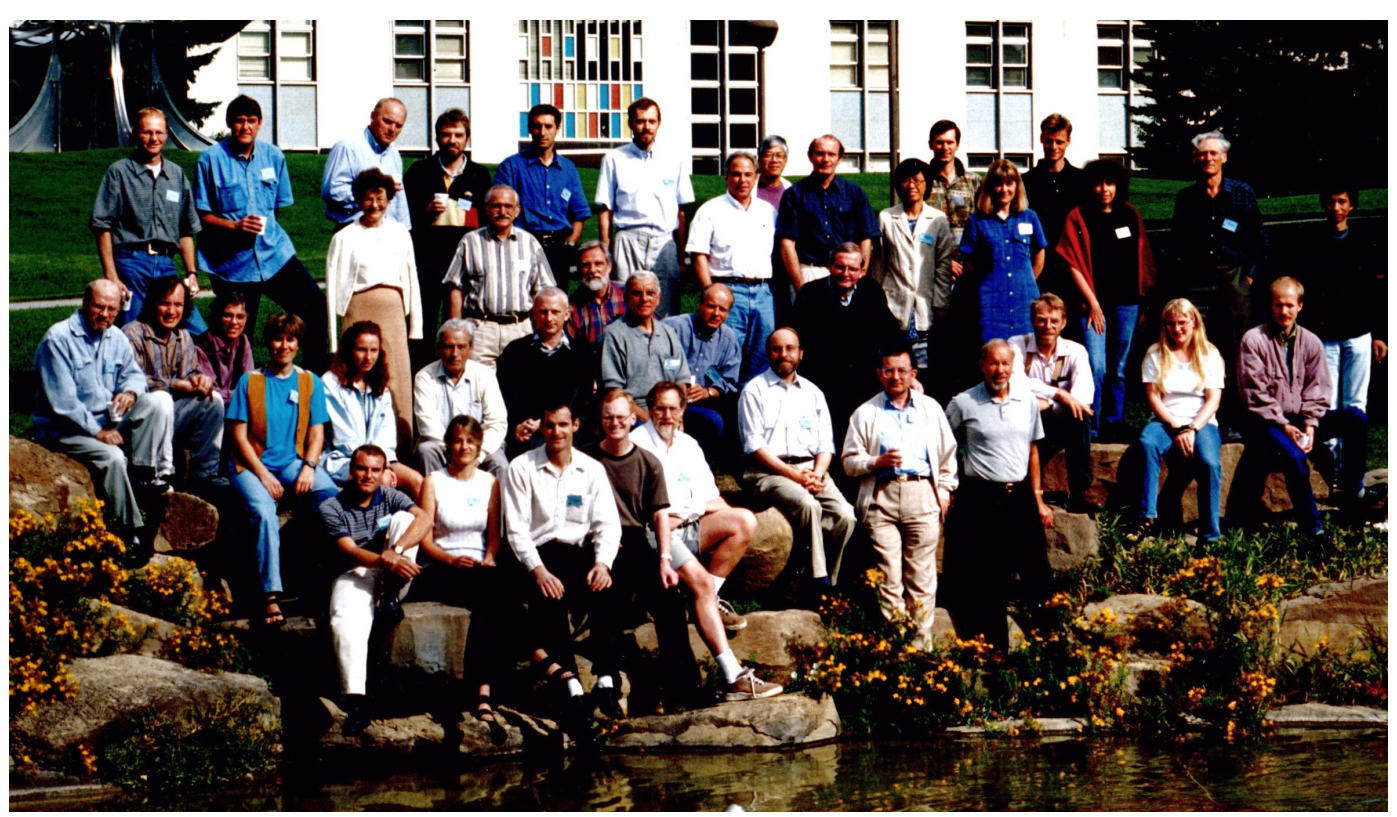

Fig. 5. A memorable group photo from the 10th Workshop on Stochastic Geometry, Stereology and Image Analysis, Calgary, 1999, organized by Ernest Enns. The social workshop arrangements reached an absolute climax at this workshop with a trip to Lake Louise and subsequent dinner, line dancing and fireworks at the country home of Ernest Enns.

geometry, spherical area measures, translative integral geometry, zonoids

- Image analysis: 3D image analysis, computed tomography, digital estimation of anisotropy, digital estimation of Euler characteristic, electron tomography, fMRI, local digital algorithms, reconstruction, scanning electron microscopy, segmentation and Markov fields, synchroton microtomography, tensor estimation

- Limit theorems for: convex hulls, convex hull peels and maximal points, distances between Poisson flats, empirical mark covariances, excursion sets, functionals of Boolean models, non-parametric intensity estimators, random graphs, random measures, random polytopes, random tessellations, shapes, $U$-statistics, Wicksell's corpuscle problem

- Particles/germ-grain models: Lilypond model, random polytopes, union of interacting disks

- Point processes: determinantal point processes, directed Markov models, Gibbs point processes, inhomogeneity, $J$-functions, Lévy driven Cox point processes, local scaling, Matérn hardcore processes with compact grains, permanent point processes, random parking, replicated point processes, stable point processes, transformation, variational analysis

- Random fields: Lévy based random fields, Markov connected component fields, polygonal Markov random fields, symmetric stable random fields
- Random graphs and networks: conductivity, distance distributions, mobility models, random walks on random graphs, spectral percolation, radial spanning tree, tessellation networks

- Random measures: invariant transport kernels, mass-stationarity, Palm measures, random surface measures, stable random measures

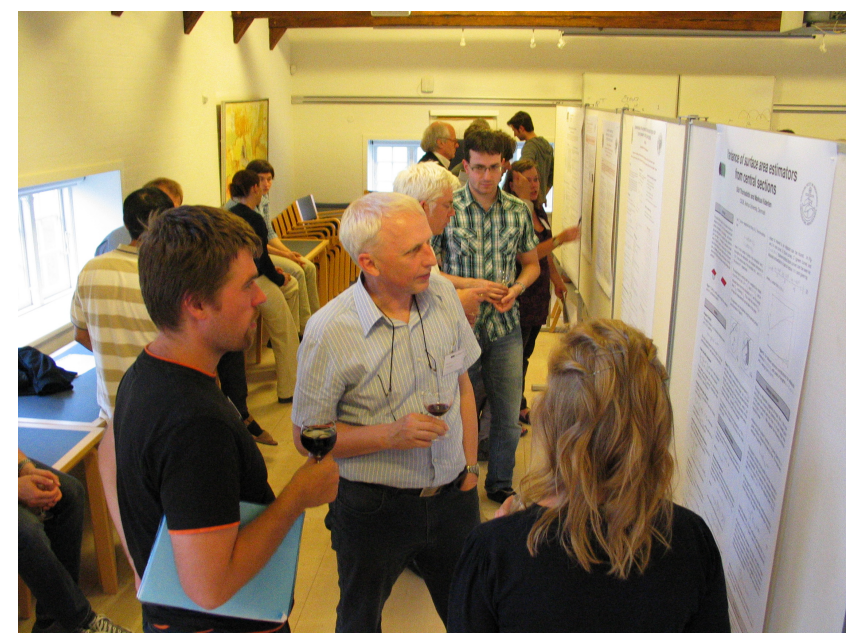

Fig. 6. From the poster session at the 16th Workshop on Stochastic Geometry, Stereology and Image Analysis, Sandbjerg, 2011, organized by Ute Hahn and Markus Kiderlen. Over the years, the number of workshop participants has increased. A large poster session with informal discussions between junior and senior researchers is now a fixed element of the workshops. 
- Random sets: anisotropy, Brownian paths, contact distributions with compact structuring element, spatial fibre and surface processes, spectral theory, Wiener sausage

- Random tessellations: anisotropy, branching random tessellations, column tessellations, gamma distributions for Poisson-Voronoi tessellations, iterated tessellations, Johnson-Mehl tessellations, multi-colour random mosaics, polyhedral Markov fields in 3D, tessellations in hyperbolic spaces, random T-tessellations, statistics of random tessellations, STIT tessellations, Voronoi tessellations with non-Poissonian point fields

- Shape: Bookstein triangle shape, PCA on shape spaces, procrustean mean shape, shape from tensors, spherical deformation model, Ziezold mean shape

- Simulation of: Boolean models, marked Gibbs processes, Markov cluster processes, nonspherical objects, operator stable random fields, polygonal Markov fields, space-time interacting discs

- Spatial statistics: Bayes inference, Bootstrap and variational approaches, envelope testing, estimating equations, estimation in Boolean models, change-set problems, estimation of the Euler characteristic, estimation of intrinsic volumes, gradient estimation, inference for inhomogeneous spatial point processes, likelihood, Palm likelihood and pseudolikelihood for point processes, moment-based estimation methods, non-parametric inference, residuals of point processes, spatial logistic regression, testing complete spatial randomness

- Stereology: asymptotics in systematic sampling, Euler number from projections, generalized Cavalieri estimator, local stereology, non-uniform systematic sampling, ratio estimation from bivariate count data, space balls, stereological estimation of size distributions for random convex polyhedrons, stereology of tensors, surfaceuniform sampling, Wicksell-type inverse problems 